\title{
Modulation of an activity response with associative and nonassociative fear in the rat: A lighting differential influences the form of defensive behavior evoked after fear conditioning
}

\author{
BILL P. GODSIL, MICHELLE A. BLACKMORE, and MICHAEL S. FANSELOW \\ University of California, Los Angeles, California
}

\begin{abstract}
Rats confronted with the onset of a light gradient display a transient increase in locomotion called the activity response (AR) and a dark preference (Godsil \& Fanselow, 2004). These experiments demonstrate that the magnitude of the AR can be blunted with Pavlovian fear-conditioning procedures via associative and nonassociative fear. Although manifested in decreased locomotion, the blunted AR effect was not due to increased freezing or immobility behaviors. Instead, rats displayed reduced rearing and an increase in a class of behaviors called stationary activity. These results suggest that the lighting differential supplied by the cue influences the topography of defensive behavior and reduces the expression of freezing. This procedure provides a means by which to examine learned and unlearned defensive responses to the same stimulus.
\end{abstract}

One view of learning emphasizes that animals have evolved a set of functional behavioral systems that act to solve naturally occurring problems (see, e.g., Timberlake, 1983). Environmental stimuli activate a functional behavior system, which then organizes behavioral strategies directed at solving the problem. This problem-oriented view focuses the analysis of animal learning on how multiple behaviors relate to each other in a manner coordinated to solve a specific challenge.

Defensive behavior is an example of a functional behavior system that has evolved in response to the selective pressure of predation. The purpose of this system is to protect animals from predation and other environmental threats. Many prey species have evolved elaborate behavioral strategies to deal with predation, and functional behavioral systems theorists provide selection rules that predict how these defensive responses are organized. For example, predatory imminence theory (Fanselow \& Lester, 1988) proposes that the selection of particular defensive responses is related to a continuum of predatory imminence that corresponds to the physical and psychological distance between the predator and the prey. Animals display preencounter defensive behaviors in situations in which a predator may be present but is undetected and postencounter defensive behaviors when a threat has been detected. In addition, these prey animals display circa-

Grants NIMH MH62122 to M.S.F. and NRSA MH12402 to B.P.G. supported this study. The authors thank M. J. Sanders and C. K. Cain for their helpful comments on the manuscript and A. Fink and R. Frescas for their help with the fear-conditioning chamber schematic. Correspondence should be addressed to M. S. Fanselow, Department of Psychology, University of California, Los Angeles, CA 90095 (e-mail: fanselow@ucla.edu). strike defensive behaviors as the attacking predator makes contact with the animal. Dark preference behavior and meal pattern adjustment are two of the rat's preencounter defensive behaviors (Godsil, Tinsley, \& Fanselow, 2003). Freezing is the rat's dominant postencounter defensive behavior (e.g., Fanselow \& Lester, 1988). Activity burst behavior, which is the vigorous unconditional locomotor response to footshock (Fanselow, 1982), as well as escape and fighting (Fanselow \& Sigmundi, 1982), are the rat's dominant circa-strike defensive behaviors.

Given this theoretical perspective, lighting conditions provide an interesting topic for the analysis of the interaction between different defensive behaviors, because light itself can evoke both unlearned and learned defensive responses. For instance, without any specific training, lighting differentials evoke a dark preference, whereby rats tend to avoid brightly illuminated places (e.g., Valle, 1970). This and other light-evoked responses are consistent with the hypothesis that higher illumination levels provide an ecologically relevant, unlearned danger signal that evokes fear (File \& Hyde, 1978; Roth \& Katz, 1979; Valle, 1970; Walker \& Davis, 1997a; Williams, 1971). In addition, with appropriate training, light cues evoke fear responses that are measures of learned fear, such as freezing and barpress suppression (e.g., Ayres, Axelrod, Mercker, Muchnik, \& Vigorito, 1985; Helmstetter \& Fanselow, 1989).

Freezing is a defensive behavior characterized by a cessation of movement that rodents display when encountering a specific threat, such as a predator or an aversive Pavlovian conditional stimulus (CS). Presumably, rats evolved freezing because of the selection pressure of predation. It protects the rodent because many predators are less likely to attack immobile targets (see, e.g., Thompson 
et al., 1981) and movement acts as a releasing stimulus for predatory attack (White \& Weeden, 1966). Freezing behavior is sensitive to a wide range of shock parameters and has been used as a behavioral index of fear learning and memory (e.g., Fanselow, 1980). In the laboratory, freezing is defined as the absence of all movement except for breathing, but this response should not be thought of as a simple inhibition of movement. Freezing is accompanied by specific physiological changes, such as shallow but rapid respiration (Hofer, 1970). Rodents typically freeze near walls or corners (Grossen \& Kelley, 1972), and they are more likely to freeze in the black compartment of a black and white colored shuttle box (Allison, Larson, \& Jensen, 1967). In addition, freezing rats show an enhanced jump (startle) to sudden stimulus change (Borszcz, Cranney, \& Leaton, 1989). Thus, freezing is not a passive response but is a component of an integrated functional defensive behavior.

We developed an open-field procedure in which a bright light gradient is presented to a rat after a dark baseline period by turning on bright light bulbs on one side of a rectangular apparatus (Godsil \& Fanselow, 2004). Rats do not freeze in response to the light gradient. Instead, during the minute after light onset, they often approach the bright end of the arena before retreating to the dark end. This sequence is often repeated, and it contributes to a transient increase in locomotion that we named the $a c$ tivity response (AR). Similar increases in locomotion are observed after lighting changes that involve (1) the termination of the light gradient (Godsil \& Fanselow, 2004), (2) the onset of the light gradient after an all-bright baseline (Godsil \& Fanselow, 2004), and (3) the onset of an all-bright condition without a light gradient after a dark baseline (Anagnostaras, Maren, \& Fanselow, 1999). Thus, the AR seems to be an exploratory behavior geared to investigating stimulus change. After the rats have explored the arena, dark preference behavior becomes prevalent.

Little is known about how learned and unlearned defensive behaviors interact. Therefore, in the present research, we examined the rat's response to a single stimulus capable of evoking both learned and unlearned defensive behaviors. To accomplish this, rats were fear conditioned with light-gradient-shock pairings, and their behavior was compared with that of control groups during a subsequent open-field test. We anticipated that after this training session, the rats would both freeze and show a dark preference in response to the presentation of the light gradient during the open-field test. In this way, the experiment examined the topography of defensive responding when a rat was confronted by a stimulus that evoked both learned and unlearned fear behaviors.

\section{EXPERIMENT 1}

\section{Method}

\section{Subjects}

Sixty female Long-Evans rats (200-325 g) were bred and raised at UCLA. The animals had free access to food and water and were kept on a 14:10-h day:night cycle. All experimental procedures occurred between 6 and $12 \mathrm{~h}$ after light onset. The subjects were group housed until they were transferred to single home cages at the start of the experiment. The experimenter handled the rats twice before the start of training and testing. Handling consisted of transporting the rats to the area adjacent to the test room and picking up each animal for approximately $10 \mathrm{sec}$.

\section{Open-Field Apparatus}

The open-field arena was a white, translucent polyethylene box (Model CB-80, Iris USA, Pleasant Prairie, WI) with internal dimensions of $69 \mathrm{~cm}$ long $\times 34 \mathrm{~cm}$ wide $\times 30 \mathrm{~cm}$ high. Black electrical tape attached to the underside of the floor marked eight squares $(17 \times 17 \mathrm{~cm})$ that were visible from above. The arena was situated on a table in the center of the room. Three lamps containing single $100-\mathrm{W}$ white light bulbs were positioned at one end of the table (see Figure 1). One lamp was positioned $14 \mathrm{~cm}$ from the center of a short wall of the rectangular arena; one lamp flanked each long side of the arena, $14 \mathrm{~cm}$ from the long walls and $15 \mathrm{~cm}$ from the same short wall. All three lamps were situated $18 \mathrm{~cm}$ above the base of the arena. When lit, the bulbs flooded one end of the open field with light (Zone 1), creating an illumination gradient across the arena. The experimenter could manipulate these lights from the adjacent room, and their luminance output could be adjusted with a dimming switch (Variac, Type W5M, General Radio Company, Concord, MA). A white noise generator provided background noise ( $65 \mathrm{~dB}$, A scale). Two cameras (directly adjacent to each other) were suspended from the ceiling directly above the center of the open field. One camera (NightShot setting, Model CCD TRV57, Sony, Japan) was used to monitor the open field while the room was dark, and another camera (Cohu, San Diego, CA) was used to monitor the arena while the three lamps illuminated the room. These cameras were connected to a monitor and VCR located in the adjacent room.

\section{Fear-Conditioning Apparatus}

The rats were fear conditioned in a chamber designed to simulate the manner in which the light stimulus would be presented on the open field (see Figure 2). This apparatus consisted of an inner chamber that was covered by the open-field chamber. The inner chamber was a transparent Plexiglas box $(58 \mathrm{~cm}$ long $\times 25 \mathrm{~cm}$ wide $\times 20 \mathrm{~cm}$ high) that was divided into three compartments along its length. The rats were confined to the center compartment (shown in gray in Figure 2 ; $28 \mathrm{~cm}$ long $\times 25 \mathrm{~cm}$ wide $\times 20 \mathrm{~cm}$ high) by two transparent

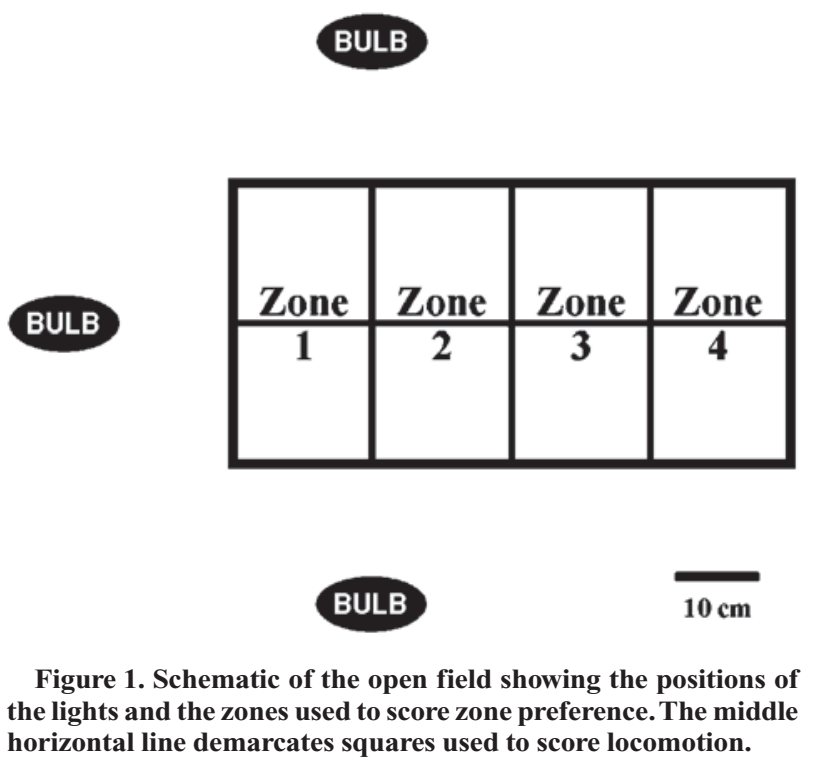
horizontal line demarcates squares used to score locomotion. 


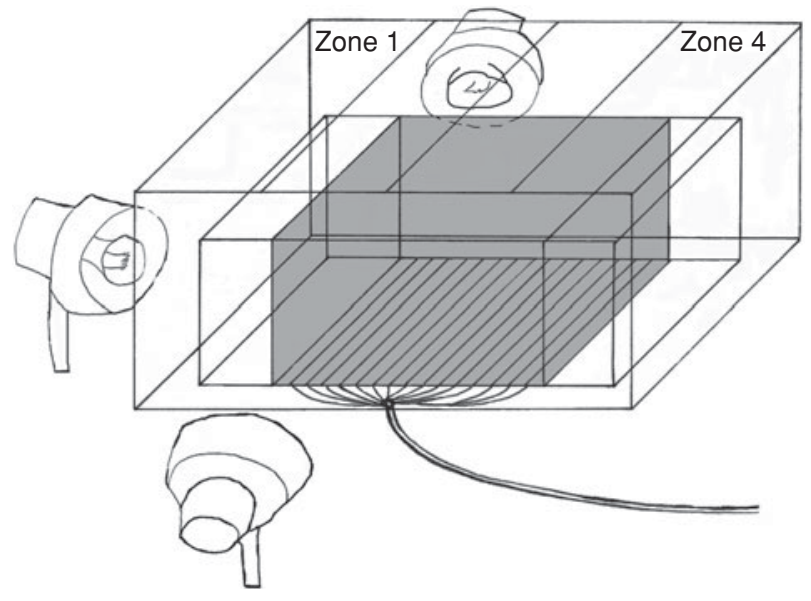

Figure 2. Schematic of the fear-conditioning chamber used in Experiment 1. The rats were confined in the middle division of a clear Plexiglas chamber with a grid floor (shown in gray). The open field (outermost chamber) was rotated upside down and placed over the Plexiglas chamber. The lights were positioned at the same distances from the open field as those used during the open-field test.

Plexiglas partitions, because only this section had an electrifiable grid floor. The 18 bars of the copper grid floor were approximately $0.38 \mathrm{~cm}$ in diameter and were spaced approximately $1.25 \mathrm{~cm}$ apart. This chamber was connected to a computer-controlled shock scrambler and generator (MED Associates, St. Albans, VT). The openfield arena was rotated upside down and positioned over the inner chamber, and three lamps were situated in a configuration identical to that of the open field: One lamp was positioned $14 \mathrm{~cm}$ from the center of a short wall of the rectangular arena; one lamp flanked each long side of the arena, $14 \mathrm{~cm}$ from the long walls and $15 \mathrm{~cm}$ from the same short wall. These lamps were positioned $18 \mathrm{~cm}$ above the base of the inner chamber. With this configuration, the lamplight passed through the translucent open-field walls, thereby mimicking how the light would later be presented during testing. The chamber was cleaned with 5\% sodium hydroxide before each test, and the collecting pan beneath the grid floor was scented with $5 \%$ benzaldehyde in alcohol before each session. The room was dark, and a fan provided background noise ( $65 \mathrm{~dB}$, A scale).

\section{Open-Field Testing}

Testing began 20 min after the animals were transported to the room adjacent to the test rooms. The rats were tested in two identical open fields. Each open field was located in a single test room. The open-field arenas were cleaned with $5 \%$ sodium hydroxide and were rotated $180^{\circ}$ before each test. The rats were tested individually in a sequence counterbalanced for group treatments. The experimenter placed the animal in the center of the open field. The 8-min test began as the door of the test room was closed. At the end of the test, the experimenter returned the subject to its cage and then cleaned the open field in preparation for the next test. For each experiment, dark phase refers to the first $4 \mathrm{~min}$, and light phase refers to the last 4 min of the test session.

\section{Light Stimuli}

Illumination levels were measured with a light meter (Model 403125, Extech Instruments, Waltham, MA). The light meter's probe sensor was situated in the center of the floor in Zone 1 or Zone 4 (with the tip of the sensor pointed at Zone 1) as the measurements were taken. In the dark condition, the open field was $0 \mathrm{Fc}$ in Zones 1 and 4. In the dim gradient condition, Zone 1 was approximately $2 \mathrm{Fc}$, and Zone 4 was approximately $1 \mathrm{Fc}$. In the bright gradient condition, Zone 1 was approximately $200 \mathrm{Fc}$, and Zone 4 was approximately $100 \mathrm{Fc}$.

\section{Behavioral Measures}

Locomotion. To provide a measure of locomotion in the open field, an observer counted the number of crossovers a rat performed during each minute of the test. A crossover was defined as a movement from square to square that included all four of the rat's paws. This measure provided an estimate of the distance traveled by each animal.

Activity response. To estimate the rat's change in locomotion in response to a change in illumination, the difference in crossovers between Minutes 4 and 5 of the test were calculated [(Minute 5 crossover count) - (Minute 4 crossover count)].

Zone preference. To estimate the time spent in each zone of the arena, zone preference was scored according to an instantaneous time-sampling procedure with which each animal was observed every $4 \mathrm{sec}$ during the 8-min test. An observer assigned a position to the rat based on an assessment of where the center of the animal's body was located (excluding the tail). These observations estimated the time that the animals spent in each zone of the arena. A zone consisted of two adjacent squares aligned vertically along the length of the arena. Thus, for this measure, the open field was divided into four equal rectangles $(34 \times 17 \mathrm{~cm}$; Figure 1). Previous studies have demonstrated that animals spend more time in Zones 1 and 4 (approximately 33\%) than in Zones 2 and 3 (approximately 17\%) when the arena is dark. This pattern reflects the rat's innate thigmotaxic tendency; Zones 1 and 4 flank the arena's corners, where thigmotaxis is normally the most robust. Given these observations, only zone preference data from Zone 4 were analyzed in these experiments.

Freezing. To estimate the percentage of time the rats spent engaging in freezing behavior, freezing was scored according to an instantaneous time-sampling procedure with which each animal was observed every 2 sec during Minute 5 of the test. Freezing was defined as the absence of movement, except that required for breathing.

\section{Minute 5 Behavioral Measures}

A behavioral inventory analysis was used to characterize rat behavior during the 5th minute of the test (previously described in Godsil \& Fanselow, 2004). Five behaviors were scored according to an instantaneous time-sampling procedure. Each animal was observed every $2 \mathrm{sec}$ during Minute 5 of the test. These observations yielded an estimate of the percentage of time spent engaging in each behavior. Grooming was defined as licking the paws or using the forepaws to wipe the face or body. Forward locomotion was defined as movements with all four limbs in a forward direction. Rearing was defined as standing on the rear limbs with the forelimbs off the ground and without movement in any lateral direction. Immobility was defined as the absence of any visible movement, except that required for sniffing, whisker movement, and breathing. Stationary activity was defined as any movement of the body that was not grooming, forward locomotion, rearing, sniffing, breathing, or whisker movement. Typically, these behaviors consisted of the following movements: (1) upper body motion that occurred as the hind limbs and body pivoted near an axis, (2) changes in posture without paw movements, (3) movements of the front paws without movement of the rest of the body, and (4) movement of the head and neck. When different observers have used this behavioral inventory procedure, they have produced similar results (reliability coefficient $=.87$ ).

\section{Procedure}

The rats were divided into six groups ( $n=10$ for each group) whose treatment differed by two factors: pairing (paired, CS only, and US only) and illumination (dim and bright). Paired groups were conditioned with light-gradient-shock pairings during the training 
session. CS-only groups were exposed to the light CS without shock during training. Unconditional stimulus (US) only groups were exposed to the shock US without light during training. Dim and bright groups were exposed to either the dim or the bright light gradient during testing. The paired and CS-only groups received the same intensity light stimulus during training and testing. The six groups were paired-dim (PD), paired-bright (PB), CS-only-dim (CD), CSonly-bright (CB), US-only-dim (UD), and US-only-bright (UB).

All the rats received the following sequence of experimental sessions: a fear-conditioning session, followed by a chamber extinction session, four open-field habituation sessions, and an open-field test. Each of these sessions was separated by a 48-h interval.

On the training day, the rats were exposed to the fear-conditioning chamber individually for $438 \mathrm{sec}$. During this single session, the rats in the paired groups (PD and $\mathrm{PB}$ ) received three light-shock pairings. For each pairing, the termination of a 16-sec light stimulus was followed by a 2-sec footshock. Light CS onset occurred 192, 274 , and $356 \mathrm{sec}$ after the rat was placed in the chamber; the shocks ( $2 \mathrm{sec}, 1.5 \mathrm{~mA}$ ) were delivered 208, 290, and $372 \mathrm{sec}$ after placement in the chamber. CS-only groups (CD and $\mathrm{CB}$ ) were presented with three light stimuli on the same schedule as that for the paired groups, but no shocks were delivered. The US-only groups (UD and UB) received three shocks on the same time schedule as that for the paired groups, but no lights were presented. The rats could not be viewed during this training, because the chamber was covered with the translucent open-field arena, so no behavioral data were collected. Two days following this training, each rat was exposed to the training chamber for $438 \mathrm{sec}$; no lights or shocks were presented during these chamber extinction sessions. These exposures were conducted in a different room, separate from both the training and the testing rooms; this step was included in order to reduce context generalization between the training and the testing chambers.

Four open-field habituation sessions occurred at 48-h intervals in the days between extinction and the open-field test. These sessions permitted the locomotion levels of all the groups to converge to a similar baseline prior to open-field testing. During a single habituation session, each rat was exposed to the open field for $8 \mathrm{~min}$. No lights were presented during these sessions. Forty-eight hours after the last habituation session, the rats were tested on the open field. Thus, the open-field test occurred 12 days after training. The test consisted of an 8-min exposure to the open field; during the first $4 \mathrm{~min}$, the room was dark, and during the last $4 \mathrm{~min}$, the open field was illuminated by the light gradient stimulus. Groups PD, CD, and UD were tested in the dark open field for $4 \mathrm{~min}$ before the dim gradient was presented for the last 4 min; Groups PB, CB, and UB were tested in the dark open field for 4 min before the bright gradient was presented during the last $4 \mathrm{~min}$. All behaviors were scored from videotape after testing. The experimenter scored these behaviors and was kept blind to the fear-conditioning and stress room lighting treatments.

\section{Statistical Analysis}

In all cases, group differences were considered to be significant when $p<.05$. Aside from the cases noted below, a factorial ANOVA was used to determine whether there were any group differences. Locomotion and zone preference data for the dark and light phases of each experiment were analyzed separately to provide measures separate from a stimulus change; these data were submitted to a repeated measures ANOVA, which included a factor for time (four 1-min blocks).

\section{Results}

After fear-conditioning and chamber extinction sessions, the rats were placed in the open field for four habituation sessions in order to ensure similar locomotion baselines prior to testing. No lights were presented during these sessions. Locomotion data from the 4th minute of each session were analyzed (with a one-way ANOVA), because this data point is used in the calculation of AR. During the first two open-field habituation sessions, the rats that had received footshock during training displayed less locomotion than did the CS-only groups [Sessions 1 and 2: group, $F(5,54)=3.22$ and 2.38, respectively; $p<$ $.05]$. However, the groups showed similar locomotion during Minute 4 of the last two habituation sessions [Sessions 3 and 4: group, $F(5,54)<1$ ]. Thus, the groups had similar locomotion baselines prior to open-field testing.

The groups showed similar locomotion during the dark phase of testing [pairing, $F(2,162)=1.48$; illumination, $F(1,162)=1.26]$, whereas during the light phase, the dim illumination groups displayed more locomotion than did the bright groups [illumination, $F(1,162)=40.07$, $p<.0001$; see Figure 3A]. The pairing treatment did not influence locomotion across the light phase interval $[F(2,162)<1]$. This pattern indicates that the bright gradient suppressed locomotion, as compared with the dim gradient.
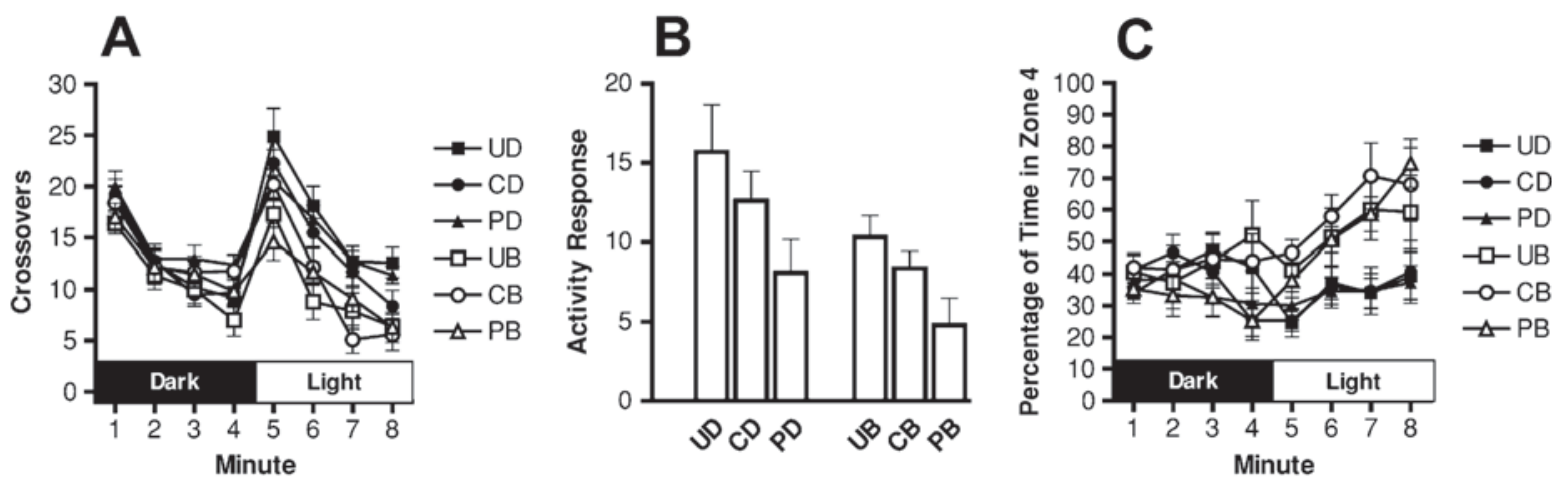

Figure 3. Experiment 1: Light-gradient-shock pairings blunt the magnitude of the activity response (AR), as compared with CS-only and US-only controls. (A) Mean locomotion estimated by the number of crossovers counted per minute. (B) Mean ARs calculated by the change in crossovers between Minutes 4 and 5 of testing. (C) Mean zone preference estimated as the percentage of time spent in Zone 4 of the open field during each minute of testing. Error bars indicate SEMs. UD, US-only dim; CD, CS-only dim; PD, paired dim; UB, US-only bright; CB, CS-only bright; PB, paired bright. 
Figure 3B contains the AR data from the open-field test. For each illumination treatment, the paired groups displayed smaller ARs than did the CS-only and US-only control groups $[F(2,54)=5.85, p<.005]$. Planned comparisons, in the form of Fisher's protected least significant difference (PLSD) test, revealed that the paired groups were different from both controls. This pattern suggests that associative fear blunted the AR behavior of the paired groups. Groups tested with the dim light gradient displayed larger ARs than did those tested with the bright gradient $[F(1,54)=7.42, p<.01]$, which indicates that the dim light gradient elicits larger ARs than does the bright light gradient.

Each group spent a similar amount of time in Zone 4 during the dark phase of the test [pairing, $F(2,162)=2.49$, $p=.092$; illumination, $F(1,162)<1$; see Figure $3 C]$. After the light gradient onset, the bright illumination groups spent more time in Zone 4 than did the dim illumination groups $[F(1,162)=27.88, p<.001]$. Fear conditioning did not influence zone preference $[F(2,126)<$ 1]. This pattern indicates that the bright gradient provoked more dark preference behavior than did the dim gradient.

To determine whether freezing caused the blunted AR to the shock-paired lights, freezing behavior was assessed during Minute 5 of testing. None of the groups displayed substantial levels of freezing ( $<2 \%$ freezing in each group). In addition, the groups did not freeze differentially [pairing, $F(2,54)<1$; illumination, $F(1,54)=1.05$ ]. Further analysis of freezing during the remainder of the test revealed a similar pattern: The groups displayed similar but low levels of freezing during Minutes 6, 7, and 8 of testing. This pattern indicates that under the present treatment conditions, light-shock pairings did not increase freezing.

The data described above suggest that learned associative fear blunts the AR without evoking substantial levels of freezing. Previous investigators have suggested that rats might display an alternative conditional response (CR) to aversive light cues that does not consist of freezing behavior (e.g., Sigmundi \& Bolles, 1983). Therefore, we conducted an inventory of the behaviors expressed during Minute 5 of the test (the minute following stimulus change) in order to better describe the rat's response to light gradient onset and to possibly identify a behavior that accounts for the blunted AR effect.

Table 1 (top) contains the Minute 5 behavioral inventory data. The rats showed very little grooming and immobility but spent more than $97 \%$ of Minute 5 engaging in forward locomotion, rearing, and stationary activity. Inspection of Minute 5 behaviors did not reveal a single behavior that was expressed more or less often by the paired groups, as compared with their controls. Instead, two trends emerged: (1) Animals that received shock during training (paired and US-only groups) tended to show more stationary activity and less rearing than did the CS only group, and (2) animals exposed to bright light also tended to show more stationary activity and less rearing than did their dim light counterparts.

\section{Discussion}

During testing, the rats in each group explored the dark open field and displayed an AR to the light gradient onset. Rats trained with light-shock pairings showed blunted ARs, as compared with their CS-only and US-only control groups. Notably, none of the rats displayed substantial levels of freezing or immobility during Minute 5 of testing. Thus, this blunted AR effect cannot be attributed to reduced locomotion due to freezing, nor can it be explained by the less restrictive measure of movement inhibition, such as the immobility measure we used. Paired rats and US-only rats did show more stationary activity than did CS-only rats, however. This pattern indicates that the expression of freezing, or immobility, is limited by sudden onset of the light cue and that rats display learned fear in a qualitatively different manner (i.e., stationary activity) when challenged by the light gradient.

The paired groups displayed blunted ARs, as compared with both CS-only and US-only controls. This pattern sug-

Table 1

Percentage of Time Expressing Each Category of Behavior During Minute 5 of Open-Field Testing in Experiments 1 and 2

\begin{tabular}{|c|c|c|c|c|c|}
\hline \multirow[b]{2}{*}{ Group } & \multicolumn{5}{|c|}{ Behavior Category } \\
\hline & Grooming & $\begin{array}{c}\text { Forward } \\
\text { Locomotion }\end{array}$ & Rearing & $\begin{array}{c}\text { Stationary } \\
\text { Activity }\end{array}$ & Immobility \\
\hline \multicolumn{6}{|c|}{ Experiment 1} \\
\hline Dim-CS-only & 0 & 43 & 43 & 13 & 2 \\
\hline Dim-US-only & 0 & 46 & 33 & 20 & 1 \\
\hline Dim-paired & 0 & 42 & 38 & 21 & 2 \\
\hline Bright-CS-only & 0 & 41 & 31 & 25 & 3 \\
\hline Bright-US-only & 0 & 39 & 28 & 31 & 2 \\
\hline Bright-paired & 0 & 39 & 21 & 38 & 1 \\
\hline \multicolumn{6}{|c|}{ Experiment 2} \\
\hline Dim-no-shock & 0 & 41 & 41 & 16 & 1 \\
\hline Dim-10-shock & 0 & 39 & 35 & 24 & 2 \\
\hline Bright-no-shock & 0 & 38 & 29 & 31 & 2 \\
\hline Bright-10-shock & 0 & 36 & 25 & 38 & 1 \\
\hline
\end{tabular}

Note-Values are rounded to the nearest integer. 
gests that nonassociative effects (pseudoconditioning and sensitization) cannot fully account for the blunted ARs and that these responses are, at least in part, the consequence of associative learning. Also, the training-to-test interval was 12 days in this experiment, which demonstrates that the blunted AR response has some temporal stability.

US-only rats showed larger ARs than did the paired and CS-only groups. This pattern likely reflects the novelty of the light gradient for the US-only group. Recall that the paired and CS-only rats were exposed to the light cue three times during the fear-conditioning procedure, whereas the US-only animals did not receive any exposure. Thus, the paired and CS-only animals may have habituated to the light gradient, which caused smaller ARs during testing. This interpretation is consistent with unpublished observations from our laboratory that indicate that the magnitude of the AR decreases with repeated exposures to the light gradient stimulus. Considering that our time-sampling procedure revealed that paired and US-only rats showed a similar frequency of forward locomotion (but different ARs), it may be that habituation to the light gradient leads to slower forward locomotion. In other words, the timesampling procedure reflects the duration of forward locomotion, whereas the crossover count that the AR is based on reflects both duration and speed of locomotion.

The rats tested with bright light displayed smaller ARs than did those tested with dim light. It seems plausible that bright light reduced the $\mathrm{AR}$, both because it reduces locomotion (e.g., Walsh \& Cummins, 1976) and because it triggers a preference for Zone 4. In addition, the rats exposed to the bright gradient displayed a stronger dark preference than did the rats exposed to the dim gradient, but fear conditioning did not influence the preference. Previous reports have shown that fear conditioning can reduce dark preference when a rat receives footshock in the dark chamber of a two-chambered light/dark box (e.g., Odling-Smee, 1975). We did not explicitly control the location of rats during our conditioning procedure; thus, shock reinforcement may not have influenced the dark preference because it was not consistently paired with a specific element of the light gradient.

\section{EXPERIMENT 2}

Experiment 1 provided evidence that learned, associative fear blunts the rat's AR to the light gradient without evoking freezing. Within these results, the performance of the US-only rats showed the largest ARs, although they showed levels of stationary activity similar to those for paired rats. This pattern may seem paradoxical, because the US-only group showed a larger increase in locomotion while simultaneously performing behaviors (with a frequency similar to that for the paired rats) that might be expected to lead to reduced locomotion (i.e., stationary activity). The purpose of Experiment 2 was to clarify the influence of nonassociative fear effects on the blunted AR response. To do so, we modified our procedure to determine (1) whether shock exposure would be suf- ficient to cause blunted ARs and increases in stationary activity, without increases in freezing or immobility, and (2) whether generalization to the lighting conditions of the training procedure would influence the ARs of shocktreated animals.

One explanation for the large ARs observed in US-only animals is that exposure to the light gradient influences subsequent responding to the stimulus (US-only rats were not exposed to the light gradient before testing, whereas paired and CS-only rats were presented with the light stimulus three times during training). Thus, previous light exposure may have caused habituation of the AR and stationary activity in the paired and CS-only groups, thereby affecting the expression of these behaviors during testing. To address concerns regarding the habituation of the AR, in Experiment 2, we compared shock and no-shock animals that were not exposed to the light gradient during training.

It is also conceivable that the US-only rats in Experiment 1 displayed large ARs because of generalization. For these animals, darkness might have signaled danger because they were conditioned with three footshocks in the dark. Considering that the open field was dark just prior to light gradient onset during testing, the light gradient might have operated as a discriminative cue that caused a large AR for US-only rats because it reduced generalized fear to darkness. To consider this possibility, we manipulated the lighting conditions during training, so that half the rats were conditioned with the room lights on and half with them off.

\section{Subjects \\ Eighty female Long-Evans rats (200-325 g) were bred, raised, and handled as in Experiment 1.}

\section{Fear-Conditioning Chambers}

The rats were conditioned in eight identical aluminum and Plexiglas chambers (aluminum sides, translucent tops and backs, transparent fronts; $28 \mathrm{~cm}$ long $\times 21 \mathrm{~cm}$ high $\times 22 \mathrm{~cm}$ deep; Lafayette Instruments, Lafayette, IN). The floors of these chambers had 18 stainless steel rods $(0.48-\mathrm{cm}$ diameter, $1.1 \mathrm{~cm}$ apart). These chambers were connected to the same shock scrambler and generator (MED Associates, St. Albans, VT) as that in Experiment 1.

\section{Open-Field Apparatus}

The same open-field apparatus as that in Experiment 1 was used.

\section{Procedure}

The rats were divided into eight groups whose treatment would differ by three factors: stress room lighting (on and off), treatment (shock and no shock), and illumination (dim and bright). Stress room refers to the room in which the rats received either 10 footshocks or no shocks during Day 1 of this experiment. On and off groups had the room lights either on or off during the shock stress session. This lighting manipulation was included to determine whether the ambient light present during shock stress would influence reactivity to light during the subsequent open-field test.

The day after the last handling procedure, the rats were exposed to a fear-conditioning chamber for $840 \mathrm{sec}$. During this training session, the shock groups received 10 unsignaled footshocks $(2 \mathrm{sec}$, 
$1.5 \mathrm{~mA}$ ), and the no-shock groups did not. The first shock occurred $192 \mathrm{sec}$ after placement in the chamber; the remaining shocks occurred at $64-\mathrm{sec}$ interstimulus intervals. No discrete light stimuli were presented during this training session. Twenty-four hours after fear conditioning, the rats were tested on the open field. Dim groups were tested in the dark open field for $4 \mathrm{~min}$ before the dim lights were illuminated for the last $4 \mathrm{~min}$; bright groups were tested in the dark open field for 4 min before the bright lights were illuminated during the last $4 \mathrm{~min}$. All behaviors were scored from the tape after testing. The experimenter scored these behaviors and was kept blind to the shock stress and stress room lighting treatments.

\section{Statistical Analysis}

Except for the cases noted, the statistical analysis followed the same method as that in Experiment 1. Again, a factorial ANOVA was used to determine whether there were any group differences. Locomotion and zone preference data for dark and light phases of each experiment were analyzed separately in order to provide measures separate from a stimulus change; these data were submitted to a repeated measures ANOVA, which included a factor of time (four 1-min blocks).

\section{Results}

This experiment was conducted in two replications. A four-way factorial ANOVA of the AR data revealed no interactions between the replication, treatment, stress room lighting, and illumination factors [all $F \mathrm{~s}(1,64)<1.9]$. In addition, stress room lighting had no discernible influence on behavior. Thus, for all subsequent comparisons, the data were collapsed across the replication and stress room lighting factors. The remaining four groups were shockbright (SB), shock-dim (SD), no-shock-bright (B), and no-shock-dim (D).

During shock stress training, freezing behavior was absent during the preshock interval. Animals belonging to the shock groups displayed a gradual acquisition of postshock freezing behavior, visible during the interstimulus intervals. Freezing was absent in the no-shock animals (data not shown).

Figure 4A contains the locomotion data from the openfield test. Groups that received footshock during training displayed less locomotion across the dark phase than did the no-shock groups [treatment, $F(1,228)=8.30$, $p<.01]$, but this difference was evident only early in the interval [treatment $\times$ time interaction, $F(3,228)=4.96$, $p<.005]$. A Fisher's PLSD post hoc test revealed that each group displayed similar levels of locomotion during Minute 4 of testing. This pattern of data indicates that the footshock treatment reduced locomotion early in the dark phase.

During the light phase, the no-shock groups showed more locomotion than did the shock groups [treatment, $F(1,228)=5.49, p<.05]$, and dim groups displayed more locomotion than did the bright groups [illumination, $[F(1,228)=22.42, p<.001]$. This pattern of data indicates that the shock stress treatment reduced locomotion and that the bright gradient suppressed locomotion to a greater extent than did the dim gradient. These effects on locomotion operated independently of each other [treatment $\times$ illumination interaction, $F(1,228)=1.16$ ].

Figure 4B contains the AR data from the open-field test. Dim illumination groups displayed larger ARs than did the bright illumination groups [illumination, $F(1,76)=8.50$, $p<.005]$. Also, groups that received shock during training exhibited blunted ARs, as compared with those that did not [treatment, $F(1,76)=6.44, p<.05]$. These effects operated independently of each other [treatment $\times$ illumination interaction, $F(1,76)<1$ ]. This pattern of data indicates that shock exposure blunted the AR, as compared with the no-shock controls, and that the bright gradient evoked a smaller AR than did the dim gradient.

Figure 4C contains the zone preference data from the open-field test. Each group spent a similar amount of time in Zone 4 during the dark phase [illumination, $F(1,228)<1$; treatment, $F(1,228)<1$ ]. The groups exposed to the bright gradient during the light phase spent more time in Zone 4 than did those exposed to the dim gradient [illumination, $F(1,228)=48.57, p<.0001$ ] The treatment variable did not influence zone preference [treatment, $F(1,228)<1$ ]. There was also a nonsignificant treatment $\times$ illumination interaction $[F(1,228)<1]$. This pattern suggests that dark preference behavior was
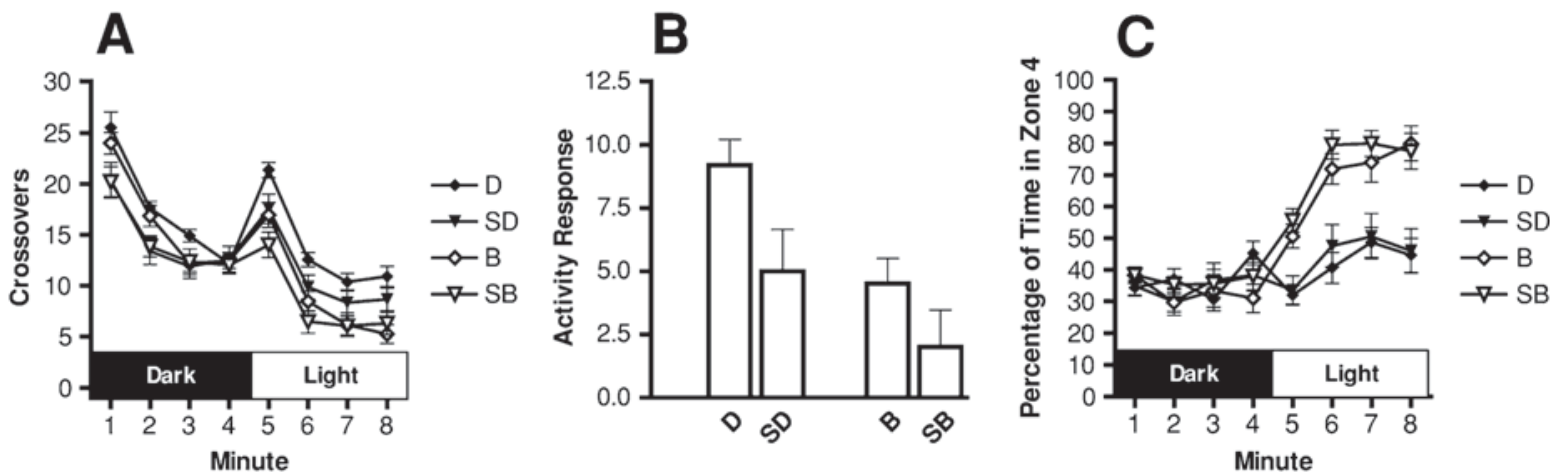

Figure 4. Experiment 2: Exposure to 10 footshocks in a chamber distinct from the open field blunts the activity response (AR). (A) Mean locomotion estimated by the number of crossovers counted per minute. (B) Mean ARs calculated by the change in crossovers between Minutes 4 and 5 of testing. (C) Mean zone preference estimated as the percentage of time spent in Zone 4 of the open field during each minute of testing. Error bars indicate SEMs. D, no-shock-dim; SD, shock-dim; B, no-shock-bright; SB, shock-bright. 
controlled by the intensity of the light gradient and that it was not influenced by the shock treatment.

Freezing behavior was assessed during Minute 5 of testing. None of the groups displayed substantial levels of freezing ( $<1.5 \%$ freezing in each group). In addition, the groups did not freeze differentially [treatment, $F(1,76)=$ 1.33 ; illumination, $F(1,76)<1$ ], nor was there an interaction between these factors. This pattern indicates that the light gradient stimuli and the Pavlovian conditioning procedures were ineffective at evoking freezing behavior just after light onset.

Table 1 (bottom) contains the Minute 5 behavioral inventory data. Much as in Experiment 1, the rats showed little grooming and immobility but spent more than $97 \%$ of Minute 5 engaging in forward locomotion, rearing, and stationary activity. As before, the animals that received shock during training or bright light during testing tended to show more stationary activity and less rearing than did their no-shock or dim light counterparts.

\section{Discussion}

The rats displayed ARs in response to light gradient onset, and those animals exposed to footshock during training displayed smaller ARs than did those that did not. This blunted AR effect was not the result of freezing or immobility displayed during Minute 5. This confirms that shock exposure alone can blunt AR and increase the expression of stationary activity without similar increases in freezing or immobility. In addition, the rats exposed to bright light displayed smaller ARs and more dark preference behavior than did those exposed to dim light.

The lighting conditions of the training procedure did not influence AR behavior. Thus, the rats did not show a tendency to generalize between the training illumination and the test light. Given that the rats were trained in a chamber dissimilar to the open field and that they had never encountered the light stimulus before, these results are consistent with the interpretation that nonassociative fear can blunt the magnitude of the AR. Presumably, the fear-conditioning treatment sensitized the rats' fear system, and they expressed this fear with blunted ARs.

It is also possible that the rats generalized between the two chambers on the basis of some other cue, such as placement in a chamber. Indeed, early in the dark phase, the shock groups displayed reduced locomotion, as compared with controls. This pattern may have been an effect of shock sensitization that was not apparent in Experiment 1 . According to this account, 10 footshocks potently sensitize the fear system, whereas 3 footshocks do not. This reduced dark phase locomotion could also be a short-term effect of shock sensitization that was not seen in Experiment 1 because of the longer training-to-testing interval and the habituation sessions. Unpublished findings from our laboratory indicate that exposure to 3 footshocks does not affect dark phase locomotion $48 \mathrm{~h}$ after training, however (Godsil, 2004). Therefore, the effect of shock on dark phase locomotion is more likely related to the number of shocks administered during training. Whatever the cause, shock and no-shock animals displayed similar locomotion during Minute 4 of the test, which provides some evidence that their generalized fear related to a placement cue or that the short-term sensitization effect had subsided.

\section{Additional Analysis}

In Experiments 1 and 2, we noted that exposure to either footshock during training or bright light during testing tended to favor the expression of stationary activity. Accordingly, we pooled the data from these experiments for an additional analysis. The rats were divided into four groups whose treatment differed by two factors: illumination (dim or bright) and shock treatment (shock or no shock). Shock or no-shock groups received footshock or no footshock prior to open-field testing. Dim and bright groups were exposed to either a dim or a bright gradient stimulus during testing. The four groups were dim-no-shock (D-NS; $n=30$ ), dim-shock (D-S; $n=$ 40 ), bright-no-shock (B-NS; $n=30$ ), and bright-shock (B-S; $n=40$ ). Group D-NS comprised Group CD from Experiment 1 and Group D from Experiment 2. Group D-S comprised Groups PD and UD from Experiment 1 and Group SD from Experiment 2. Group B-NS comprised Group CB from Experiment 1 and Group B from Experiment 2. Group B-S comprised Groups PB and UB from Experiment 1 and Group SB from Experiment 2.

A three-way ANOVA with between-group factors for experiment (Experiment 1 or 2), illumination (dim or bright), and treatment (shock or no shock) revealed no interaction between the factors. Consequently, for all subsequent analyses, these groups were pooled to assess the influence of light and shock on Minute 5 behaviors.

The rats exposed to the bright gradient showed less forward locomotion $[F(1,136)=4.06]$ and rearing $[F(1,136)=34.99]$ but more stationary activity $[F(1,136)=35.84]$ than did their dim counterparts. The rats exposed to shock during training displayed less rearing $[F(1,136)=8.01]$ and more stationary activity $[F(1,136)=9.20]$ than did their no-shock counterparts. This pattern of results indicates that previous shock stress and/or bright lights during testing favor the expression of stationary activity. These findings are consistent with those of previous work that has shown that rats display increased rearing to a dim, localizable light source (Holland, 1977).

\section{GENERAL DISCUSSION}

Rats confronted with a sudden onset of a light gradient display a transient increase in locomotion. These experiments demonstrate that the magnitude of this response can be blunted with fear conditioning to the light and that the reduced locomotion is not due to freezing behavior. With appropriate training, the blunted AR response has properties consistent with its being a CR to the aversive light CS. Namely, rats trained with light-shock pairings displayed smaller ARs than did their nonassociative controls, and this effect persisted 12 days. Thus, this procedure may be 
useful for investigations of associative learning. Rats that received extensive exposure to footshock also displayed a blunted AR, which demonstrates that our open-field task is sensitive to nonassociative fear manipulations and may provide a useful test for generalized anxiety.

Rats often display freezing to aversive Pavlovian CSs and a dark preference to lighting differentials. For the paired animals in Experiment 1, the light gradient was an aversive CS that provided a lighting differential. Given this stimulus complexity, one might expect the paired rats to display a combination of these defensive behaviors; yet they showed very little freezing. The low levels of freezing observed in the blunted AR response is interesting because freezing has been described as the rat's dominant defensive behavior for shock-paired CSs (Fanselow \& Lester, 1988) and the freezing response is sensitive to a wide range of conditioning parameters (e.g., Fanselow, 1980).

We suggest that the lighting differential exaggerates the expression of behaviors that are incompatible with freezing. The light's onset produces a substantial change in illumination and a lighting differential that extends across the test arena. Under these circumstances, stationary activity, rearing, and other exploratory behavior (i.e., forward locomotion) are prominent. Importantly, the lighting differential does not simply disrupt conditional responding but, instead, alters the form of the CR. Given that freezing is not prevalent and other defensive behaviors do emerge, this procedure may represent a situation in which the lighting differential shapes the topography of defensive responding, with exploration, dark preference, and stationary activity behaviors occupying most of the response.

Generalization decrement may also contribute to the low level of freezing seen in the paired groups. These rats were confined to a small Plexiglas shock box covered by the open-field chamber during training, whereas they were tested in the open field. This arrangement might alter the quality of light or influence how rats perceive the light cue. Thus, although the blunted ARs indicate that the rats learned a light-shock association, our procedure may produce levels of fear beneath the threshold that evokes freezing.

Our results demonstrate that the expression of stationary activity is positively correlated with brighter light intensity and with previous shock exposure. We suggest that stationary activity is a set of exploratory behaviors that supplant rearing in situations with increased danger or predatory imminence. They may serve a similar function as rearing (i.e., exploration), but stationary activity may be a degraded or more cautious form of exploration. Interestingly, stationary activity behaviors often involve body postures that position the rat close to the ground, and they resemble the stretched approach behavior previously characterized as a fear response (e.g., Blanchard, Yang, Li, Garvacio, \& Blanchard, 2001; Fanselow, 1994).

One might also conceptualize stationary activity as being part of a graded continuum in which freezing represents the most extreme form of movement inhibition (i.e., freezing $>$ immobility $>$ stationary activity $>$ locomotion). According to this analysis, the light gradient may cause a shift on the movement inhibition continuum that favors the expression of stationary activity over freezing. Further experiments will be needed to substantiate this hypothesis. Our data indicate that stationary activity replaces rearing, without any increases in immobility or freezing.

Several reports have indicated that light CSs tend to evoke less freezing behavior than do tone CSs (e.g., Ayres et al., 1985; Helmstetter \& Fanselow, 1989; Sigmundi \& Bolles, 1983). One interpretation of reduced freezing is that a light CS might elicit a CR other than freezing, yet this other CR to light has not been detected (see Albert \& Ayres, 1997). We observed that fear conditioning increased the expression of stationary activity. This pattern suggests that rats show a set of behaviors conditioned by shock, although no singular other CR might exist. Instead, rats engage in a repertoire of behaviors that serve a similar exploratory function, whose form may differ substantially. This pattern of results resembles those of Gibson (1952) who noted that goats readily acquire leg flexion as a CR. Importantly, this action was a common component for a set of responses to the aversive Pavlovian CS, such as walking forward, walking backward, and wheeling or circling. Thus, the blunted AR may result from the expression of a set of behaviors that all contribute to decreased locomotion.

In Experiment 1, the US-only group showed a larger AR than did the CS-only and paired groups, whereas in Experiment 2 the US-only groups (the shock groups) showed blunted ARs, as compared with unshocked controls. We have suggested that this pattern was related to habituation of the AR and stationary activity that occurred during the training phase of Experiment 1. An alternative account of our data asserts that under some conditions ( 3 unsignaled shocks with extensive open-field chamber habituation), shock exposure facilitates the AR (see Williams, 1972) and that, under other conditions (10 unsignaled shocks), shock exposure blunts the AR. Experiment 1 did not include the untreated control group (one that received neither shock nor light prior to testing), however, and therefore we can neither reject nor accept the hypothesis that mild shock facilitates the AR.

The fear-conditioning procedures did not influence dark preference behavior. Odling-Smee (1975) demonstrated the dark preference could be reduced by fear conditioning rats in the dark compartment of a two-chambered apparatus. In contrast, we did not control the rat's location during training; thus, it is possible that the rats received shock in both the dark and the bright ends of the light gradient. In addition, Blanchard et al. (2001) have argued that avoidance responses, such as the dark preference, are biologically "cheap" (meaning that animals expend little energy performing this response). This interpretation suggests that rats will readily express avoidance responses if they have no need to approach the potentially threatening area. Thus, dark preference behavior may be less sensitive to manipulation, because rats express it readily. 
Previously, we reported that rats display an AR to the sudden onset of a bright gradient (Godsil \& Fanselow, 2004). Here, we have described experiments in which both bright and dim light gradients were used. Obviously, the absolute intensity of the light, as well as the light gradient (differential between the dark and the light areas), differs for the dim and the bright conditions. Different components of the rat's behavior seem to be related to the absolute level of light and to the gradient itself. First, higher illumination evokes a more robust dark preference. Second, the dim gradient evokes a larger AR than does the bright gradient. Third, rats display more rearing to the dim gradient, whereas the bright gradient favors the expression of more stationary activity behaviors. These features are consistent with the hypothesis that higher illumination evokes fear and suppresses locomotion, whereas stimulus change evokes locomotion and rearing that might reflect exploratory activity. Whereas the tendency to explore novel stimuli causes the rat to approach a light that is suddenly turned on, its aversiveness causes the rat to approach the darkest portion of the apparatus. The behavior the animal exhibits is a compromise between these tendencies. Therefore, the behavioral richness of this preparation provides a means to simultaneously assess these behavioral tendencies under conditions that are designed to manipulate such factors as anxiety.

In our open-field task, the blunted AR effect is a measure of learned fear, and dark preference behavior is a measure of unlearned fear. Thus, this task provides a means to assess learned and unlearned fear responses to the same light stimulus in the same test session. This procedure can provide another approach for teasing apart the anatomy of fear learning and anxiety (e.g., Walker \& Davis, 1997b).

\section{REFERENCES}

Albert, M., \& Ayres, J. J. B. (1997). One-trial simultaneous and backward excitatory fear conditioning in rats: Lick suppression, freezing, and rearing to CS compounds and their elements. Animal Learning \& Behavior, 25, 210-220.

Allison, J., Larson, D., \& Jensen, D. D. (1967). Acquired fear, brightness preference, and one-way shuttlebox performance. Psychonomic Science, 8, 269-270.

Anagnostaras, S. G., Maren, S., \& Fanselow, M. S. (1999). Temporally graded retrograde amnesia of contextual fear after hippocampal damage in rats: Within-subjects examination. Journal of Neuroscience, 19, 1106-1114.

Ayres, J. J. B., Axelrod, H., Mercker, E., Muchnik, F., \& Vigorito, M. (1985). Concurrent observations of barpress suppression and freezing: Effects of CS modality and on-line vs. off-line training upon posttrial behavior. Animal Learning \& Behavior, 13, 44-50.

Blanchard, R. J., Yang, M., Li, C., Garvacio, A., \& Blanchard, D. C. (2001). Cue and context conditioning of defensive behaviors to cat odor stimuli. Neuroscience \& Biobehavioral Reviews, 25, 587-595.

Borszcz, G. S., Cranney, J., \& Leaton, R. N. (1989). Influence of longterm sensitization on long-term habituation of the acoustic startle response in rats: Central gray lesions, preexposure, and extinction. Journal of Experimental Psychology: Animal Behavior Processes, 15, 54-64.

FANSELOW, M. S. (1980). Conditioned and unconditional components of post-shock freezing. Pavlovian Journal of Biological Science, 15, 177-182.

FANSELOW, M. S. (1982). The postshock activity burst. Animal Learning \& Behavior, 10, 448-454.

FANSELOW, M. S. (1994). Neural organization of the defensive behavior system responsible for fear. Psychonomic Bulletin \& Review, 1, 429-438.
Fanselow, M. S., \& Lester, L. S. (1988). A functional behavioristic approach to aversively motivated behavior: Predatory imminence as a determinant of the topography of defensive behavior. In R. C. Bolles \& M. D. Beecher (Eds.), Evolution and learning (pp. 185-211). Hillsdale, NJ: Erlbaum.

FanSELOW, M. S., \& Sigmundi, R. A. (1982). The enhancement and reduction of defensive fighting by naloxone pretreatment. Physiological Psychology, 10, 313-316.

File, S. E., \& Hyde, J. R. (1978). Can social interaction be used to measure anxiety? British Journal of Pharmacology, 62, 19-24.

GiBSON, E. (1952). The role of shock in reinforcement. Journal of Comparative \& Physiological Psychology, 45, 18-30.

GoDsIL, B. P. (2004). Defensive reactions to bright light in the rat (Doctoral dissertation, University of California, Los Angeles, 2003). Dissertation Abstracts International, 64, 5375.

GodsiL, B. P., \& FANSELOW, M. S. (2004). Light stimulus change evokes an activity response in the rat. Learning \& Behavior, 32, 299-310.

Godsil, B. P., Tinsley, M., \& Fanselow, M. S. (2003). Motivation. In I. B. Weiner (Series Ed.) \& A. F. Healy \& R. W. Proctor (Vol. Eds.), Handbook of psychology: Vol. 4. Experimental psychology (pp. 3360). New York: Wiley.

Grossen, N. E., \& Kelley, M. J. (1972). Species-specific behavior and acquisition of avoidance behavior in rats. Journal of Comparative \& Physiological Psychology, 81, 307-310.

Helmstetter, F. J., \& FAnselow, M. S. (1989). Differential secondorder aversive conditioning using contextual stimuli. Animal Learning \& Behavior, 17, 205-212.

HofER, M. A. (1970). Cardiac and respiratory function during sudden prolonged immobility in wild rodents. Psychosomatic Medicine, 32, 633-647.

Holland, P. C. (1977). Conditioned stimulus as a determinant of the form of the Pavlovian conditioned response. Journal of Experimental Psychology: Animal Behavior Processes, 3, 77-104.

ODLING-SMEe, F. J. (1975). The role of background stimuli during Pavlovian conditioning. Quarterly Journal of Experimental Psychology, 27, 201-209.

Roth, K. A., \& Katz, R. J. (1979). Stress, behavioral arousal, and openfield activity: A reexamination of emotionality in the rat. Neuroscience \& Biobehavioral Reviews, 3, 247-263.

Sigmundi, R. A., \& Bolles, R. C. (1983). CS modality, context conditioning, and conditioned freezing. Animal Learning \& Behavior, 11, 205-212.

Thompson, R. K. R., Foltin, R. W., Boylan, R. J., Sweet, A., Graves, C. A., \& Lowitz, C. E. (1981). Tonic immobility in Japanese quail can reduce the probability of sustained attack by cats. Animal Learning \& Behavior, 9, 145-149.

TimberlaKe, W. (1983). The functional organization of appetitive behavior: Behavior systems and learning. In M. D. Zeiler \& P. Harzem (Eds.), Advances in the analysis of behavior: Vol. 3. Biological factors in learning (pp. 177-221). Chichester, U.K.: Wiley.

VALLE, F. P. (1970). Effects of strain, sex, and illumination on open-field behavior of rats. American Journal of Psychology, 83, 103-111.

WALKer, D. L., \& Davis, M. (1997a). Anxiogenic effects of high illumination levels assessed with the acoustic startle response in rats. Biological Psychiatry, 42, 461-471.

WALKER, D. L., \& DAVIS, M. (1997b). Double dissociation between the involvement of the bed nucleus of the stria terminalis and the central nucleus of the amygdala in startle increases produced by conditioned versus unconditioned fear. Journal of Neuroscience, 17, 9375-9383.

Walsh, R. N., \& Cummins, R. A. (1976). The open-field test: A critical review. Psychological Bulletin, 83, 482-504.

White, C. M., \& WEEDEN, R. B. (1966). Hunting methods of gyrfalcons and behavior of their prey (ptarmigan). The Condor, 68, 517-519.

WiLliams, D. I. (1971). Maze exploration in the rat under different levels of illumination. Animal Behaviour, 19, 365-367.

WiLLIAMS, D. I. (1972). Effects of electric shock on exploratory behaviour in the rat. Quarterly Journal of Experimental Psychology, 24, 544-546.

(Manuscript received December 3, 2003; revision accepted for publication January 17, 2005.) 